\title{
Novel Experimental Method for Microscale Contact Analysis in Composite Fabric Forming
}

\author{
O. Smerdova ${ }^{1,2} \cdot$ M.P.F. Sutcliffe ${ }^{1}$
}

Received: 18 December 2014 / Accepted: 20 May 2015 / Published online: 3 June 2015

(C) The Author(s) 2015. This article is published with open access at Springerlink.com

\begin{abstract}
This paper describes a novel experimental rig and associated experimental method developed to investigate composite fabric/tool contact at the microscopic scale. A key feature of this method is that it enables direct observation of real contact at the scale of fibres and the evolution of this contact under simultaneous application of shear and compression loads. To observe the contact, an optical semi-reflective coating is used. An algorithm is developed to analyse the contact images and measure the real contact length and orientation of individual fibres. The method is applied to microcontacts of carbon twill fabric. The real contact length under an apparent pressure of $1.9 \mathrm{kPa}$ is surprisingly small compared to the apparent contact length. Transverse forces associated with friction are also measured. However these results are difficult to interpret as the test generates friction forces which differ from those which would be seen in conventional sliding friction tests.
\end{abstract}

Keywords Carbon fibre $\cdot$ Fabrics/Textiles ·

Micro-mechanics $\cdot$ Forming

M. P. F. Sutcliffe

mpfs@eng.cam.ac.uk

O. Smerdova

olga.smerdova@ensma.fr

1 Department of Engineering, University of Cambridge, Trumpington Street, Cambridge CB2 1PZ, UK

2 Present address: Institute P', Department of Physics and Mechanics of Materials, ENSMA - CNRS - University of Poitiers, 1 avenue Clément Ader, 86961 Futuroscope Chasseneuil cedex, France

\section{Introduction}

Shear is the principal deformation mode of composite fabrics while being formed into a curved shape [1]. Therefore, knowing fabric shear characteristics is vital for modelling of the forming of composite parts. These characteristics are usually obtained either experimentally [2-7] or by virtual tests $[8,9]$. Benchmark forming experiments of dome and double-dome shapes revealed that the local shear angle varies by tens of degrees within the component [1]. There are two principal experimental methods to study shear of composite fabrics: picture frame and bias extension tests [2]. While the former results in a rather uniform strain field in a rhombus-shaped fabric specimen, the latter provides three different shear deformation zones in the rectangular specimen. The shear angle dependence on the applied force is measured in both tests and used to deduce fabric shear characteristics which can serve as input parameters in numerical models. The results are rather difficult to interpret and often differ between two tests, and even between the same test carried out on an identical textile in different laboratories [7].

Although shear deformation in real composite forming is usually accompanied by a compression load, both of the above-mentioned tests operate with shear loading alone. The combination of both loadings might change shear load-displacement behaviour. This issue has been addressed very recently in experimental work $[10,11]$ presenting a device capable of applying combined tension, shear and lateral pressure on the fabric specimen, and in a finite element model of composite forming [12]. Unlike these studies focusing on macroscopic and mesoscopic fabric behaviour, the current paper presents a novel experimental rig designed to investigate fabric behaviour at the microscale under simultaneous shear and compression loading. A major aim of this work is to provide a tool for measurement of the contact conditions at the local scale and hence assist in modelling and understanding of friction phenomena. 
The surface geometry of the composite fabric changes significantly under shear in picture frame tests, as well as during the draping of a dome shape, as has been revealed by microscopic observations [13, 14]. It is reasonable then to suppose that friction would be influenced by this continuous surface change. Indeed, the local fibre/tool and fibre/fibre contacts at the microscale, as well as the whole contact pattern at the mesoscale, constantly evolve under shear loading. The sum of these micro-contacts form the real contact area of the fabric/ tool contact.

Knowing the real contact area is necessary to understand and model accurately friction in composite fabric forming. For example a generally accepted model of adhesive friction for sliding of solids holds that frictional force is directly proportional to real contact area [15]. Alternative models of the frictional behaviour would also require, as a prerequisite, knowledge of the contact details. The real contact area can be measured by optical, electrical, acoustic and surface-coating methods [16]. Each of these methods presents some limitations, such as transparency of one or both of the bodies, electrical conductivity requirements or high sensitivity to surface films and impurities. One of the optical methods, based on the relation between the colour of an interference fringe and the distance from the contact, was chosen and adapted to measure the fabric/tool real contact area in the current work as described below.

The optical interference method is usually used to measure the thickness of a lubricant layer in the hydro-dynamic or elasto-hydro-dynamic lubrication regime [17-20]. In this context, an oil-lubricated contact between a steel ball and glass disc under rolling, sliding or combined tribological conditions is investigated. This method is based on observation and measurement of the interference fringes formed between a part of the light beam reflected from the bottom side of the glass disc and the other part of this beam reflected from the steel ball surface. The colour and order of interference fringes correspond to local lubricant film thickness in a manner which can be calibrated to give quantitative measurements.

In order to enhance the interference fringe visibility, the bottom surface of the glass disc is often coated with a semireflective chromium layer [20]. The thickness of this layer must be optimised to provide equal reflectance and transmittance so as to produce clear interference fringes. This technique has several limitations, for example the minimum thickness that can be measured using visible light is around 45$75 \mathrm{~nm}$ [21]. An important advance to overcome this issue and to measure ultra-thin lubrication films made use of an additional $\mathrm{SiO}_{2}$ spacer layer deposited on top of the Cr layer [18]. The thickness of this layer must also be optimized depending on the type of contact being observed.

The current paper combines a mechanical loading stage similar in principle but not identical to those used in the composite forming research community - with an optical interference method used in the tribological community for observing real contact area. Therefore, this novel device enables measurements necessary to understand contact phenomena in composite forming. It is anticipated that, as with other tribological systems, a modelling approach could then be adopted, based on this physical insight, to tackle the complexities of friction generation within the fabric and at the interfaces between fabric and tool. The focus of this paper is a description of this new experiment and its mechanical and optical analysis. An image analysis algorithm developed for identifying the real contact area from the derived images is also presented. Further work in [22] adopts the methodology described in this paper and develops a multi-scale contact area analysis to characterise the contact conditions.

\section{Materials \& Methods}

\section{Experimental Equipment}

A micromechanical experimental rig has been developed to investigate composite forming behaviour at the microscopic scale. The small size and shape of the rig allow it to be fitted under the microscope. A key feature of this rig is its capability of applying simultaneously shear and compression loadings.

As shown in Fig. 1, the rig includes two miniature linear motors (Newport MFA-CC) mounted on a $1 \mathrm{~cm}$ thick aluminium plate. An open shear frame is specially designed for this experiment to hold the composite specimen, connect it to the motors and apply shear deformation. Both parts of the frame sit on aluminium pins of diameter $1.5 \mathrm{~mm}$ with a clearance so that they can rotate freely around the pin. The fibre bundles in a $5 \mathrm{~mm}$ margin around the edge of the specimen are fixed with sticky tape prior to these fabric edges being clamped into the shear frame. The clamping force is applied via screws passing through the shear frame arms and the fabric. As the motors move apart, the shear frame continuously closes, gradually increasing the shear angle of the fabric as shown in Fig. 2.

The compression force $N$ normal to the plane of the fabric is applied with two symmetrically placed screw-spring-nut systems. Two glass plates of $20 \mathrm{~mm}$ width compress the fabric sample in the central area. The dependence between the applied force and the spring length was measured with a FlexiForce ${ }^{\circledR}$ pressure sensor placed between two glass plates prior to the experiments. This relationship was found to be linear with a stiffness of $0.79 \mathrm{~N} / \mathrm{mm}$. The spring deformation was consequently used as an indication of the compression force during the experiment when composite fabric is sheared between the glass plates. This force is assumed to only vary due to a change in fabric thickness. Moreover, a rectangular slot in the aluminium support block restricts movement of the glass plates in the direction of the motor motion.

The transverse force is measured by two load cells mounted on top of each motor and connected to the shear frame. The load cells (model MLP-10, Transducer Techniques) are 
Fig. 1 Experimental rig: (a) photograph; (b) deformation schema

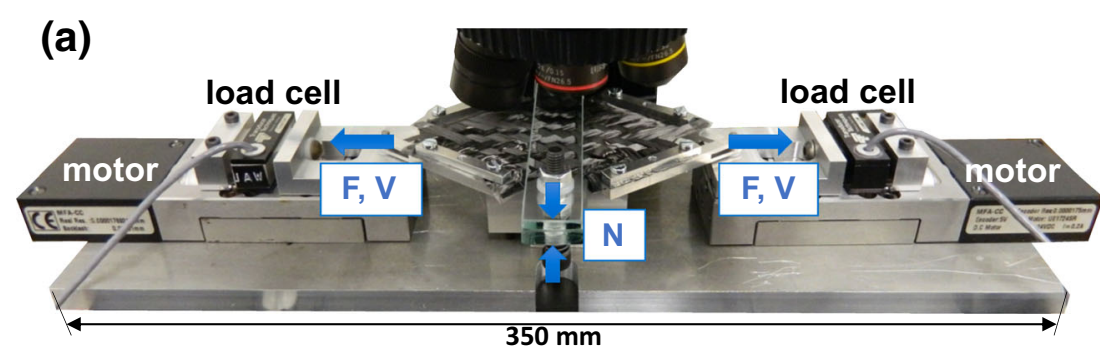

(b)

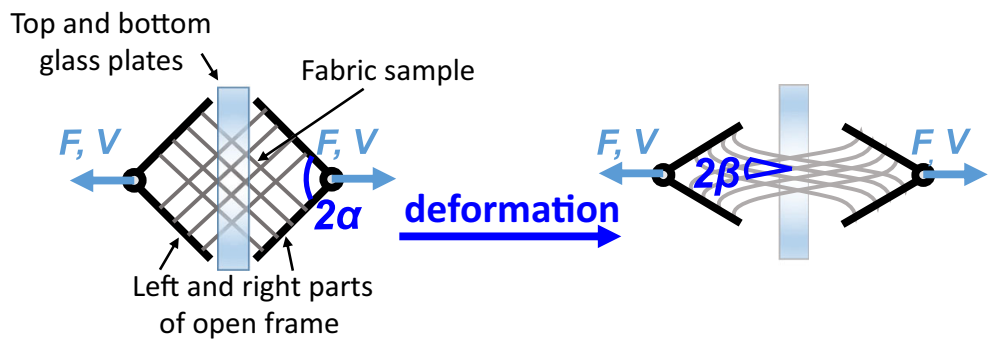

connected through a full bridge strain gauge amplifier to a PC computer via a data acquisition device (NI USB-6009).

The whole assembly is placed under an Olympus BX $51 \mathrm{M}$ microscope equipped with a digital camera (Altra 20 ) with a resolution of $1196 \times 1600$ pixels in order to observe the deformation and sliding of the fabric specimen under transverse loading. With the lens of magnification $\times 5$ used in this study, each pixel has a side length of $1.515 \mu \mathrm{m}$. A linear stage (Prior ProScan II) installed in place of the microscope table is used to translate the whole rig horizontally, while vertical movement is controlled by a motor to allow auto-focussing.

\section{Material}

The equipment described in the previous section can be used to analyse fabric/tool contact deformations of a composite ply of any architecture and material. However, because the optical coating was optimized to the reflective index of carbon fibres, the real contact area observation with this coating would be possible only on carbon fabric materials. A $2 \times 2$ twill woven T700 (C24k) carbon fibre fabric of areal weight $400 \mathrm{gsm}$ and without binder was used in the experiments presented below. Firstly, a square sample of area $80 \times 80 \mathrm{~mm}^{2}$ is cut from a fabric roll. Then, all bundles further than $20 \mathrm{~mm}$ away from the sample centre are removed. The central square woven area of $40 \times$ $40 \mathrm{~mm}^{2}$ is compressed between the glass plates and analysed under the microscope. This removal of tows away from the centre of the sample is done to minimise the friction between tows outside the gauge section (see Fig. 2). The ends of the fibre bundles are clamped in the open shear frame such that the bundles are parallel to the sides of the frame.
Fig. 2 Composite fabric deformation at times $0,10,18$ and $25 \mathrm{~s}$ after the start of frame movement. Angles $2 \alpha$ and $2 \beta$ are defined in the left top image
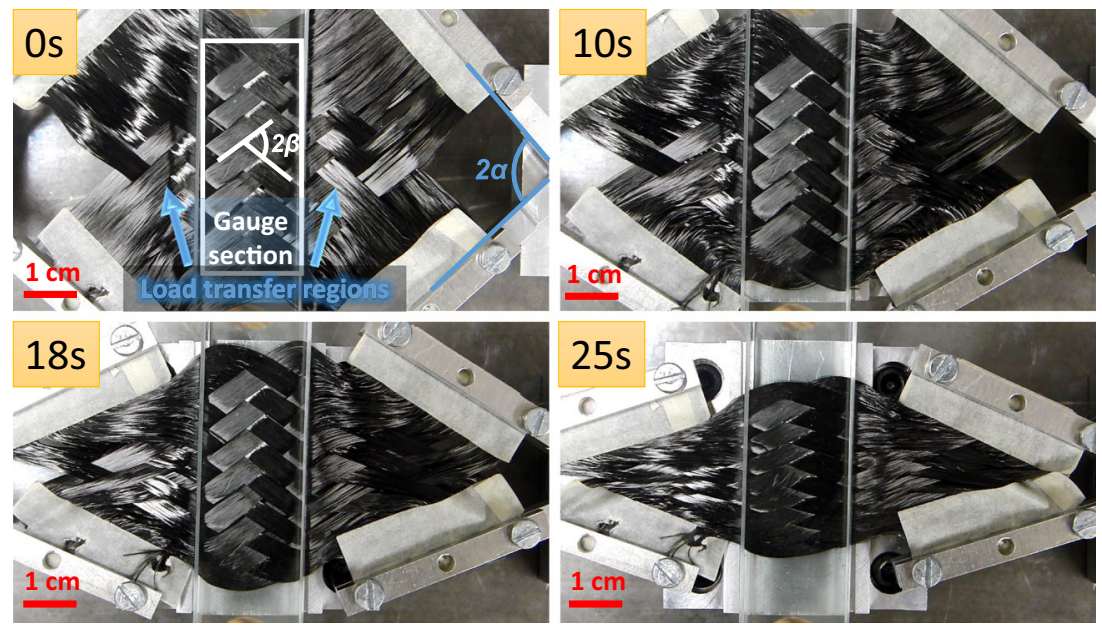


\section{Contact Area Measurement Method}

\section{Imaging method}

To make use of the optical method presented in the Introduction to investigate the contact, one of the glass plates was covered with a semi-reflective coating (Tofico, France). The layer thickness of the semi-reflective coating must be adjusted depending on the reflectivity of the observed object to give the best colour contrast between the contact and non-contact areas. Since carbon fibre fabric is the object of our study, the optical coating thickness was adjusted to clearly observe carbon fibre contacts. A stack of one chromium and one silica coating layer was chosen to observe the smallest distances between the fabric fibres and the plate. The thicknesses of both layers were optimized for carbon fibre contact visualisation using TFcalc software [23] at the LTDS laboratory of the Ecole Centrale de Lyon in France. The optimization was made for an incident angle of $0^{\circ}$, white light and with the reference wavelength of $633 \mathrm{~nm}$. Using the optical characteristics of carbon fibres and both layers and taking into account the type of deposition method, the optimal values were found to be $8 \mathrm{~nm}$ for chromium and $140 \mathrm{~nm}$ for silica. Although the coating with these thicknesses has worked well in the results presented here, one needs to be cautious when repeating this experiment. To obtain the required optical properties, i.e., reflectance and transmittance equal to 0.3 , the coating thickness needs to be corrected according to the energetic parameters of the deposition method which influences the density of atomic packing and porosity and, therefore, the optical properties of the coating.

In theory, this optical contact observation method does not present significant limitations on contact size. In practice, the limits of the detected contact size are imposed by the microscope camera resolution and the observation area. Moreover, there is no limitation on the carbon fibre material architecture, although only the contact of the carbon fibres can be seen clearly using this specific coating, so that for example binder or polymer stitches present in non-crimp fabric would not be clearly visible. In principle contact of other optically reflective fibres could be observed if the coating thickness were adjusted to take into account the fibre's reflectivity.

\section{Image analysis algorithm}

An example of the image of the carbon fibre fabric is shown in Fig. 3. The fibres in contact and the interference fringes along these fibres can be easily distinguished in Fig. 3(a), indicating areas closer and farther from the contact. For the sake of this analysis, only the brightest areas which are in direct contact with the glass are considered.

In order to detect these areas automatically, a Matlab [24] algorithm has been developed as presented in Fig. 4. The fibre
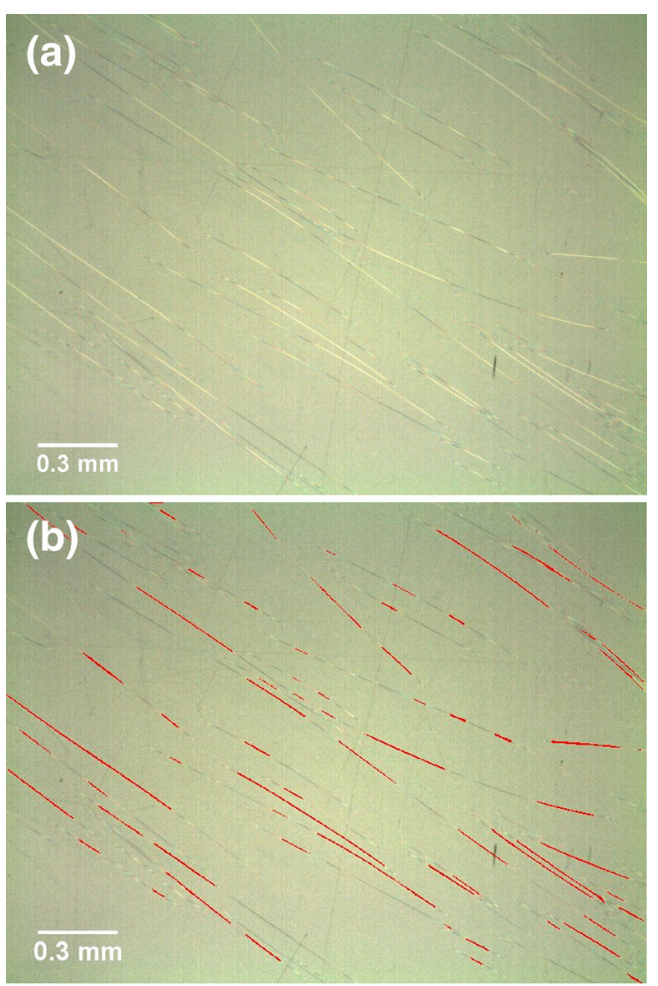

Fig. 3 Microscopic image of fibre contacts with interference fringes (a) and corresponding resulting image of the Matlab fibre detection algorithm (b). The area captured in the image is $1.8 \times 2.4 \mathrm{~mm}$ (magnification $\mathrm{x} 5$ )

contacts are detected with the aid of a filter scanning the original image searching for long and thin objects as described below.

Before the main analysis, the microscope RGB image is converted to greyscale and the non-uniform background due to the light source is removed. Then, a square $41-$ by- 41 pixel filter is created with ones in a 3-pixel-wide horizontal band in the middle and zeroes elsewhere. The size of this filter is one of the parameters of the algorithm that has been tuned to obtain best results in terms of fibre detection and noise minimisation. The whole microscopic contact image is convolved with this filter to give an image matrix in which high pixel intensities correspond to fibre contacts which are orientated horizontally (i.e., parallel to the filter). Then, the white band in the filter is rotated by $1^{\circ}$ and the analysis is repeated to obtain another image highlighting objects at an orientation of $1^{\circ}$. This process is repeated with the filter rotated between 0 and $90^{\circ}$ in steps of $1^{\circ}$. The result of this analysis is a set of 91 , with each image identifying fibres at an angle between 0 and $90^{\circ}$.

When all orientations have been considered, an intensity threshold is applied to all 91 images to detect the areas in contact. In order to eliminate possible noise and surface impurities that can interfere with the contact identification, a further filter is applied to detect only long and thin objects by imposing a threshold value of 0.99 on the eccentricity of all contacts. 
Fig. 4 Image analysis algorithm for identifying fibres in contact

\author{
1. Convert RGB to \\ gray \& remove non- \\ uniform background \\ colour
}
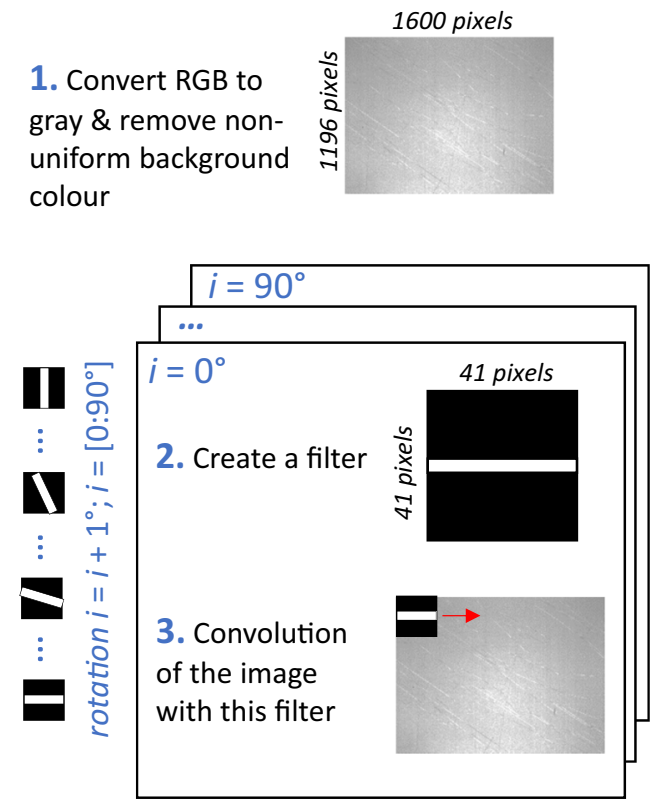

4. Obtain a stack of 91 images with highlighted fibres

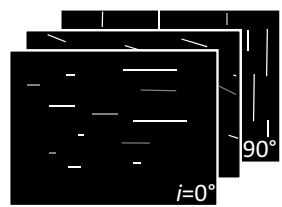
5. Apply intensity threshold to all images \& filter noise

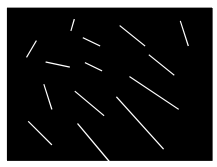

Double contacts? (visual image check)

6. Apply higher intensity filter for no these areas

7. Measure length and orientation of each fibre contact
Since some fibres form double- or triple-fibre contacts which are seen in the image as thicker areas, these areas are reconsidered a second time with a higher intensity threshold to separate individual fibre contacts. Finally, the length and the orientation of each fibre contact in the image are measured.

\section{Results \& Discussions}

\section{Image Analysis of Microscopic Contact of Twill Carbon Fabric}

Figure 3(b) shows the result of the image analysis algorithm implemented on the microscopic image shows in Fig. 3(a). The detected fibre contacts are highlighted in red. A comparison of the two images in Fig. 3 shows that all contacts discernible to the naked eye are effectively captured by the algorithm adopted despite the presence of some scratches and impurities seen on the original image. The details of the image analysis algorithm were optimised in an ad hoc way to ensure that the clearlyvisible fibres were effectively captured. To verify the robustness of the chosen parameters, the effect was examined of changing two key variables, the filter length and the threshold for bright regions. Results for a 'reference case' for a filter length of 41 pixels and a threshold of 14 were compared with calculations for changing the filter length to 31 , or changing the threshold values to 10 or 18 . These threshold values were chosen as being on the limit of credibility when compared with the visual check. Averaging the fibre length calculated over 72 images, there was negligible difference for the change in filter length, and $\mathrm{a}+13$ and $-20 \%$ change in fibre length for changes in brightness threshold. These results are considered acceptable, representing extreme ranges for the estimated fibre contact length and demonstrating the robustness of the algorithm. Additional data related to this publication is available at the Cambridge University data repository (http://www.repository.cam. ac.uk/handle/1810/247243).

Although the length of linear contact of all fibres can be measured by this algorithm rather accurately, the width of the contact patches cannot be measured due to limitations of image resolution. Therefore, this width should be estimated or measured by other methods, as discussed in [22].

Figure 5 presents part of an image of a twill carbon fabric contact region composed of several collated images with magnification $\times 5$. This region is chosen to illustrate the real contact length of one tow contact and the space between adjacent tows.

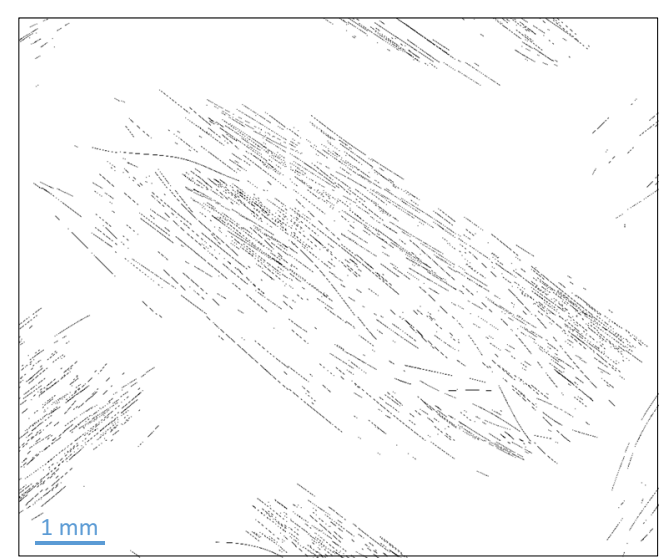

Fig. 5 Real area of contact for one whole tow at the beginning of the first test with a compressive load of $1.77 \mathrm{~N}$ 
This image is taken under a normal load of $1.77 \mathrm{~N}$, corresponding to a normal pressure of $1.9 \mathrm{kPa}$. The number of fibres in contact is rather low compared to the 24,000 fibres in the yarn. These contacts are intermittent and the orientation of many fibres differ significantly from the overall orientation of the tow. The real tow contact is rather sparse and the distance between adjacent tows is large compared to these contacts, forming a surprisingly low real contact area compared to the apparent contact (i.e., the whole area of the image in this case).

The methods described in this paper are used and developed in [22] to perform a multi-scale quantitative analysis of the real contact area on a large number of images of a given sample. The variability within samples can then be used to assess the accuracy of the measurement.

\section{Analysis of the Geometry and Loads Applied by the Rig}

The analysis presented below characterizes the geometry and loading of the current test procedure in order to help to position the current test relative to the two classical shear tests. Indeed, the loading applied by the new experimental rig is partly similar to the picture frame test, where the shear is applied by a frame, and partly like the bias extension test, where the shear angle is different in the central and edge zones. A shear experiment without compression and one under a compression load of $1.77 \mathrm{~N}$ were carried out to perform this analysis. Transverse loads at both ends of the sample were continuously measured with a sampling frequency of $1 \mathrm{kHz}$. The deformation of the whole fabric specimen was observed with a digital camera (Casio EX-FH20) with a resolution of $1280 \times 720$ pixels. In order to analyse shear deformation, a video of the experiment was recorded with a frame rate of 29 frames/sec.
Fabric shear deformation and open shear frame angles

The angle $2 \alpha$ between the two arms of each part of the shear frame and the angle $2 \beta$ between the crossing carbon fabric bundles (as shown in Fig. 2) were measured from the video images recorded during the experiment. The orientations of six tows are considered for each deformation step to calculate the average value and standard deviation of $2 \beta$. The results for the shearing experiments without compression and with a compressive load of $1.77 \mathrm{~N}$ are presented in Fig. 6.

In the no-compression shear test, the rate of angle decrease is slightly faster for the composite bundles than for the shear frame. This might be due to the initial difference between $2 \alpha$ and $2 \beta$, which is about $20^{\circ}$. Previous work $[3,25]$ using digital image correlation measurements at the mesoscopic scale show that, while the principal mode of deformation is shear in the test section in bias extension or picture frame tests, the composite fabric tows also undergo rigid body motion. This was found to be the case until the locking angle was reached. Then the lateral compression between the yarns led to a fast increase of the shear force. They conclude that "the strain field within the yarn is null or very weak". Although the mesoscopic behaviour of the tows under shear loading alone has not been characterised in our test, we believe that it is likely to be similar to the behaviour observed in the classic fabric shearing experiments. On the other hand, the compression loading and friction between fabric and tool may affect this rigid-tow behaviour.

For the case where the fabric is compressed, Fig. 6 shows that the fabric shear angle in the central clamped area remains unchanged for about the first $16 \mathrm{~s}$ of the test and then decreases rapidly. The extension during this initial phase of the test serves to deform the relatively few bundles that have not been removed from the region outside the clamped area, as
Fig. 6 Experimentally measured angles between grips $2 \alpha$ and between bundles $2 \beta$

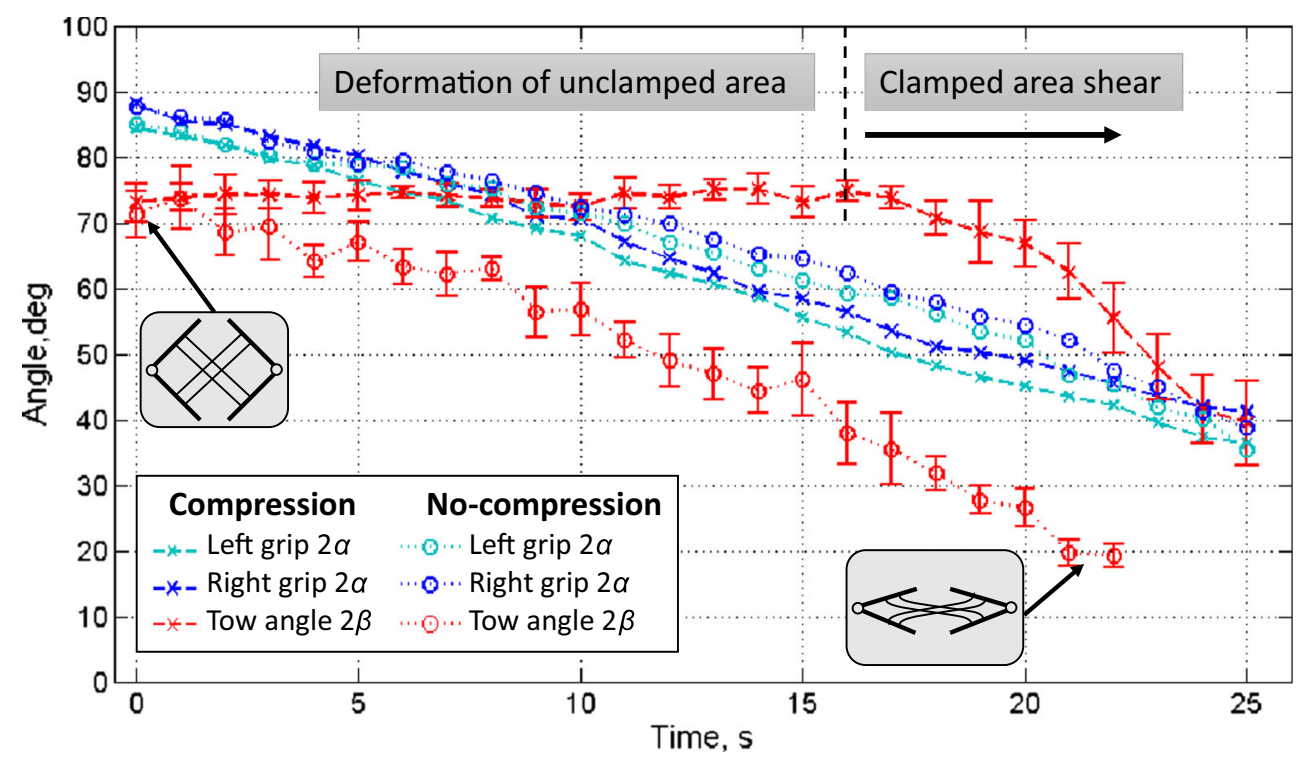




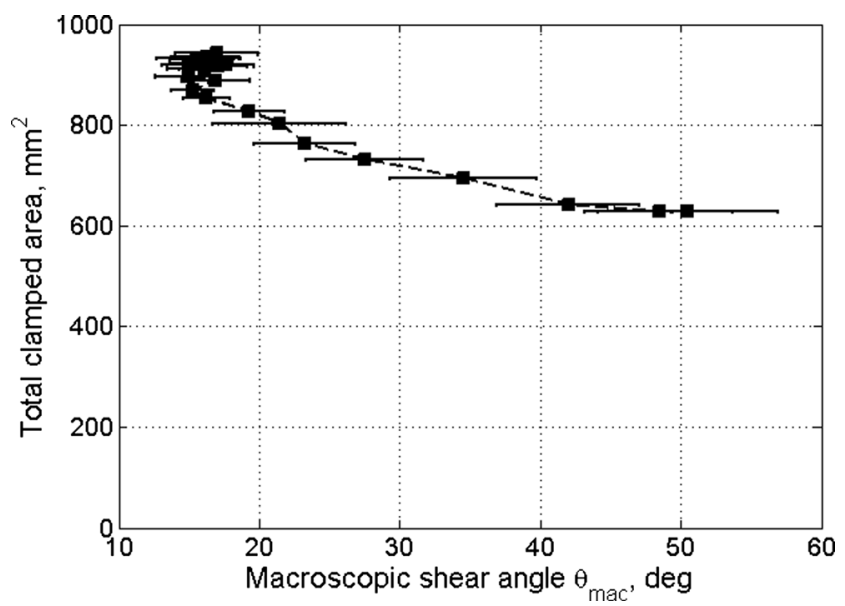

Fig. 7 Macro-effect of decreasing apparent contact area with increasing shear angle

shown in the sequence of four images seen in Fig. 2. As in the classical picture frame test, clamping of fabric in the grips results in local fibre bending close to the grips. At the end of this initial phase, the outermost sections of the bundles are highly curved and reach a locking angle limit. However the details of the deformation of these outer bundles are not critical, as the role of these regions is only to transmit a force which causes deformation of the gauge section, which in this case begins to shear $16 \mathrm{~s}$ into the test.

There is a slight discrepancy of about $5^{\circ}$ between the angle $2 \alpha$ of the two parts of the open frame for both tests. This is due to asymmetry in the way that the specimen is fixed into the grips. A similar problem can occur in the picture frame test, where differences in sample fixation, clamping forces, pretension and fabric misalignment lead to a lack of reproducibility in the results [7]. Misalignment of individual yarns also contributes to unsymmetrical loading both in the picture frame test $[6,26]$ and in the proposed compressed open-frame shear test.

\section{Apparent contact area, normal pressure and friction force}

The width of the apparent contact area, i.e., the whole sample area clamped between the glass plates, falls as the specimen elongates during the test. This change in width, which is measured using video imaging, is plotted in Fig. 7 as a function of macroscopic shear angle. While the shear angle increases from 15 to $50^{\circ}$, the total clamped area drops by around $35 \%$. On the other hand, the change of sample thickness due to lateral shrinking is about $0.4 \mathrm{~mm}$ between the start and the end of experiment. This corresponds to an increase of normal force of about $23 \%$, from 1.77 to $2.17 \mathrm{~N}$. The apparent normal pressure $p_{a p p}$ increases gradually during the experiment due to these changes in normal force and apparent contact area, rising by about $89 \%$ from the start to the end of the experiment.

A comparison of the measured transverse forces with and without compression is presented in Fig. 8. The difference between the measured force curves might be attributed to the friction between the fabric and two glass plates. However, caution should be used with this interpretation because there is a difference between the usual "sliding friction" and the friction measured in the current experiments, which might be termed "shearing friction". Sliding friction is associated with relative movement between two solid bodies. It is characterised by a sliding friction force, which is a macroscopic characteristic neglecting internal deformations of the sliding body. The friction force measured in the current experiments
Fig. 8 Transverse force evolution with time and dependence on macroscopic shear angle with either a $1.77 \mathrm{~N}$ compressive force or no compression of the fabric
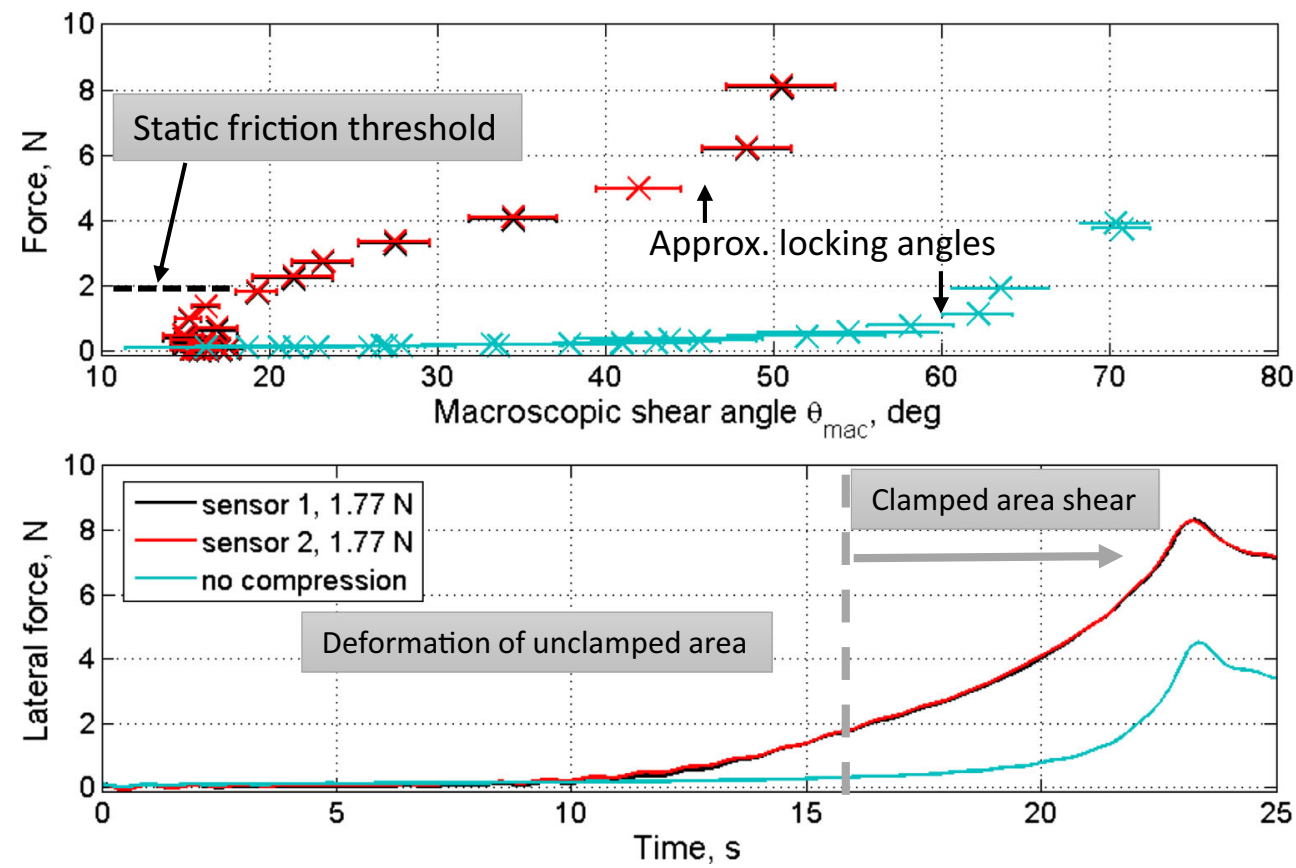
is a combination of the internal friction between carbon fibre bundles and friction between carbon fibre bundles and the two glass plates. Both these phenomena are due to shearing of the composite fabric, rather than a rigid body macroscopic sliding of the fabric as a whole relative to the glass plates.

The shearing experiment without compression, presented in Fig. 8, shows that the frictional forces between the carbon bundles are rather small for shear angles less than the locking angle, which is estimated as about $60^{\circ}$. Considering the dependence shown in Fig. 8 of the shear angle in the compressed central section on the applied shear force, it appears that the shear angle starts to change when a compression force of around $2 \mathrm{~N}$ is applied. Taking into account the two contacts on both sides of the fabric, this threshold applied force corresponds to a static shearing friction coefficient of 0.57 . The further growth of transverse load with increasing shear angle is a combination of kinetic shearing friction forces, increased inter-bundle interaction forces approaching the locking angle and traction forces. The increase of contact pressure due to sample thickening may also contribute to the increase of the transverse force.

While the experimental setup enables transversal force measurement, this data is not the main focus of the study. The friction of fibres compressed by a forming tool in a sheared 3D composite is very complex and its investigation should be approached in stages. Firstly, the measurement of contact area between the fabric and tool using the rig presented in this paper gives access to information about local fibre deformations and movements close to the surface. This real contact area allows calculation of the real contact pressure which can form the basis for accurate micromechanical modelling of fabric deformation in the forming processes. Then, the contact between fibres and bundles inside the fabric will need to be investigated, since an accurate fabric forming model would need an understanding of both tool/fabric and bundle/bundle contacts at the microscopic scale.

\section{Conclusions and Future Work}

The novel experimental rig and methods presented in this paper allow for the first time the observation of the true contact area between carbon fibre fabric and a smooth tool. The methods have been successfully validated on a twill carbon fabric. An optical coating of optimized thickness on the compressing glass plates allows observation of the carbon fabric/glass contacts at the microscopic level. A set of image analysis algorithms has been developed to provide automated detection of individual fibre contacts in the microscopic images and measurement of their length and orientation. In conjunction with the observations, the mechanical loading provided by the rig applies shear deformation to the gauge section of the fabric in the range of shear angles relevant to fabric forming processes. This shear of the fabric might be expected to change the contact conditions and hence the tribology of the contact.

The measured dependence of the applied transverse force on the shear angle follows a similar trend to those seen to traditional shear experiments using the picture frame or bias extension tests. However, the interpretation of the measured forces is less obvious. The addition of a normal compression load on the fabric creates additional friction forces between bundles within the fabric and between the fabric and the compressing plate which contribute to the measured force. With the focus of the rig on observation of the microscopic contact, issues over interpretation of the measured forces is not seen as a critical limitation, although further work would be needed to use the rig to derive useful friction measurements.

Acknowledgments The authors are very grateful to Nazario Morgado and Dr Juliette Cayer-Barrioz from the LTDS laboratory in Lyon (France) for the calculations of the optical properties of semi reflective coatings, and to the Tribology Group of Imperial College London for help with these coatings. We also acknowledge the contribution of our industrial partners Jaguar Land Rover and Granta Design Ltd, as well as the academic partners from the Composites group at the University of Nottingham. This work was funded by an Engineering and Physical Sciences Research Council grant (reference EP/K032798/1).

Open Access This article is distributed under the terms of the Creative Commons Attribution 4.0 International License (http://creativecommons.org/licenses/by/4.0/), which permits unrestricted use, distribution, and reproduction in any medium, provided you give appropriate credit to the original author(s) and the source, provide a link to the Creative Commons license, and indicate if changes were made.

\section{References}

1. Long AC (2007) Composites forming technologies. Woodhead, Cambridge

2. Harrison P, Clifford MJ, Long AC (2004) Shear characterisation of viscous woven textile composites: a comparison between picture frame and bias extension experiments. Compos Sci Technol 64: $1453-1465$

3. Lomov SV, Willems A, Verpoest I, Zhu Y, Barburski M, Stoilova T (2006) Picture frame test of woven composite reinforcements with a full-field strain registration. Text Res J 76:243-252

4. Nestor TA, Bradaigh CMO (1995) Experimental investigation of the intraply shear mechanism in thermoplastic composites sheetforming. Key Eng Mater 99-100:19-36

5. Stanley WF, Mallon PJ (2006) Intraply shear characterisation of a fibre reinforced thermoplastic composite. Compos A Appl Sci 37: 939-948

6. Hivet G, Duong AV (2011) A contribution to the analysis of the intrinsic shear behavior of fabrics. J Compos Mater 45:695-716

7. Cao J, Akkerman R, Boisse P et al (2008) Characterization of mechanical behavior of woven fabrics: experimental methods and benchmark results. Compos A Appl Sci 39:1037-1053

8. Verpoest I, Lomov SV (2005) Virtual textile composites software WiseTex: integration with micro-mechanical, permeability and structural analysis. Compos Sci Technol 65:2563-2574

9. Blacklock M, Bale H, Begley M, Cox B (2012) Generating virtual textile composite specimens using statistical data from micro- 
computed tomography: 1D tow representations for the binary model. J Mech Phys Solids 60:451-470

10. Böhler P, Härtel F, Middendorf P (2013) Identification of forming limits for unidirectional carbon textiles in reality and mesoscopic simulation. Key Eng Mater 554-557:423-432

11. Härtel F, Böhler P, Middendorf P (2014) An integral mesoscopic material characterization approach. Key Eng Mater 611-612:280-291

12. Döbrich O, Gereke T, Cherif C (2014) Modelling and simulation of the coupling of normal pressure and shear force in plain woven fabrics. Key Eng Mater 611-612:363-367

13. Chang SH, Sutcliffe MPF, Sharma SB (2004) Microscopic investigation of tow geometry changes in a woven prepreg material during draping and consolidation. Compos Sci Technol 64:1701-1707

14. Chang SH, Sharma SB, Sutcliffe MPF (2003) Microscopic investigation of tow geometry of a dry satin weave fabric during deformation. Compos Sci Technol 63:99-111

15. Bowden FP, Tabor D (2001) The friction and lubrication of solids. Oxford University Press, Oxford

16. Bhushan B (2010) Modern tribology handbook, two volume set, CRC Press

17. Cameron A, Gohar R (1966) Theoretical and experimental studies of the oil film in lubricated point contact. Proc R Soc Lond A291: $520-536$
18. Cann PM, Spikes HA, Hutchinson J (1996) The development of a spacer layer imaging method (SLIM) for mapping elastohydrodynamic contacts. Tribol Trans 39:915-921

19. Foord CA, Hammann WC, Cameron A (1968) Evaluation of lubricants using optical elastohydrodynamics. ASLE Trans 11:31-43

20. Foord CA, Wedeven LD, Westlake FJ, Cameron A (1969) Optical elastohydrodynamics. Proc Inst Mech Eng 184:487505

21. Spikes HA, Cann PM (2001) The development and application of the spacer layer imaging method for measuring lubricant film thickness. Proc Inst Mech Eng J215:261-277

22. Smerdova O, Sutcliffe MPF (2015) Multiscale tool-fabric contact observation and analysis for composite fabric forming. Compos Part A Appl Sci 116-124-124410.1016/j.compositesa.2015.03.009

23. http://www.sspectra.com/, accessed 19th Sept 2014

24. http://www.mathworks.fr/products/matlab/, accessed 19th Sept 2014

25. Lomov SV, Boisse P, Deluycker E, Morestin F, Vanclooster K, Vandepitte D, Verpoest I, Willems A (2008) Full-field strain measurements in textile deformability studies. Compos A Appl Sci 39: $1232-1244$

26. Willems A, Lomov SV, Verpoest I, Vandepitte D (2009) Drapeability characterization of textile composite reinforcements using digital image correlation. Opt Lasers Eng 47:343-351 